\title{
Capture of kinesthesis by a competing cutaneous input
}

\author{
George H. Van Doorn • Jakob Hohwy • \\ Mark A. Symmons
}

Published online: 3 June 2012

(C) Psychonomic Society, Inc. 2012

\begin{abstract}
In four experiments, blindfolded participants were presented with pairs of stimuli simultaneously, one to each index finger. Participants moved one index finger, which was presented with cutaneous and/or kinesthetic stimuli, and this movement caused a raised line to move underneath the other, stationary index finger in a yoked manner. The stimuli were $180^{\circ}$ rotations of each other (e.g., $<$ and $>$ ), and thus when a $<$ was traced with the moving finger, it caused a $>$ to be felt at the stationary finger. When asked to report the experience, participants predominantly reported the cutaneous stimulus, seemingly being ignorant of the kinesthetic stimulus. This appears to be an intrahaptic capture phenomenon, which is of interest because it suggests that conflict between intrahaptic sensory stimuli can go unnoticed; sometimes we are unaware of how we moved, and sometimes we do not know what we touched. The results are interpreted in light of optimal integration, perceptual suppression, reafference suppression, and inattentional blindness.
\end{abstract}

Keywords Haptics $\cdot$ Attention $\cdot$ Perception and action

Sensory cues can interact in a number of ways, depending on their sensory modalities, spatiotemporal properties, and whether they are consistent or contradictory. Attention, perceptual thresholds, and cue reliability also play roles. The

G. H. Van Doorn $(\varangle) \cdot$ M. A. Symmons

School of Applied Media and Social Sciences, Monash University,

Churchill 3842, Australia

e-mail: george.vandoorn@monash.edu

\section{J. Hohwy}

Department of Philosophy, Monash University,

Clayton 3800, Australia two, or more, sensory cues may be blended to form a single percept, but with one modality having relatively more influence on the percept than another. A strong case of this is capture, which implies total dominance (i.e., one sensory cue having complete influence on a bimodal percept), such as the visual capture of the location of auditory stimuli seen in paradigmatic cases of the ventriloquist effect (Pick, Warren, \& Hay, 1969). A sensory cue can also suppress another cue entirely, as in the Colavita effect, where participants often fail to respond to suprathreshold auditory stimuli when they are simultaneously presented with visual stimuli, despite an ability to respond to these stimuli when they are presented alone (Colavita, 1974; Sinnett, Spence, \& Soto-Faraco, 2007).

Suppression can also happen intramodally, as in the interocular case of flash suppression, where a light flashed during a saccade is not seen (Dodge, 1900). A similar effect (i.e., saccadic suppression of displacement) occurs when the location of a target is changed during a saccade and is not noticed. Ziat, Hayward, Chapman, Ernst, and Lenay (2010) showed that a similar effect exists in haptics - namely, tactile suppression of displacement - and provided evidence suggesting that the effect is not due to movement "but to the brief interruption of sensory input when a feature is in the gap between two fingers" (p. 301).

Little is known about integration mechanisms for scenarios in which moving objects interact with observers who are themselves moving. Some studies have suggested a degree of visual capture of moving auditory stimuli by apparent visual motion (Sanabria, Soto-Faraco, \& Spence, 2004). Seno, Ito, and Sunaga (2011) reported that the illusory perception of self-motion usually induced by purely visual stimuli (i.e., vection) is facilitated by self-generated locomotion on a treadmill. Calabro, Soto-Faraco, and Vaina (2011) showed that detection of visual movement is crossmodally facilitated by auditory stimuli. However, a large 
part of everyday, dynamic interactions with the causes of sensory cues occurs through haptics, and very little is known about the extent to which there is perceptual capture and suppression in this sensory modality.

An influential framework for understanding capture is optimal Bayesian cue integration (Ernst, 2004; van Beers, Wolpert, \& Haggard, 2002). Important work has been done on visuoproprioceptive (e.g., Van Beers et al., 2002) and visuohaptic (e.g., Ernst \& Banks, 2002) integration, arguing that they obey maximum likelihood estimation. However, only a few studies have examined intrahaptic perceptual capture and suppression - that is, such mechanisms occurring within or between haptic submodalities, which we here understand to be kinesthesis (including proprioception), and cutaneous or tactile inputs that generally arise from contact between the skin and a surface.

Very recently, Pritchett, Gallace, and Spence (2011) showed that change blindness exists in the tactile modality, although it is not as pervasive as in the visual modality. Their participants were aware of tactile change but were not aware of the location of the change (i.e., observers retained some tactile information). Another recent experiment demonstrated that although perception of traversed distance was possible using touch alone, the cutaneous discrimination threshold was larger than that of kinesthesis alone (Bergmann Tiest, van der Hoff, \& Kappers, 2011). Furthermore, cue combination relied predominantly on kinesthetic information, a finding similar to that reported by Pritchett et al. (2011), in that there was no obvious connection to optimal cue integration. Juravle and Spence (2011) showed that goal-directed movement leads to decreased tactile sensitivity and to more conservative criteria for detecting tactile changes. Questions remain regarding the strength and direction of capture in the haptic domain and the roles of attention and stimulus type. Furthermore, within the haptic domain new types of questions arise, because haptic cue integration could be subject to differences between passively guided and active movements.

Answers to some of these questions are being sought here in an exploration of a case of intrahaptic capture. Previously, Symmons, Richardson, and Wuillemin (2007) used a mechanical device to simultaneously present a kinesthetic + cutaneous experience to one hand and a cutaneous-only stimulus to the other in the exploration of raised-line letter and number stimuli. Both hands travelled the same movement pathway over the same symmetrical stimuli, but the stimuli were $180^{\circ}$ rotations of each other. For example, one hand explored a raised-line letter "p," while the other was presented with a "d." Symmons et al. (2007) found that explorers sometimes reported what they were feeling under one finger, while at other times they reported what was present under the other finger. That is, on occasion, kinesthesis dominated cutaneous information, while at other times the reverse occurred - cutaneous capture of kinesthesis. The authors argued that this effect depended on how much cutaneous and kinesthetic information was present at each finger. However, the most interesting finding was that participants did not notice that the dominant submodality's stimulus was different from the stimulus being explored by the suppressed, or captured, submodality. The responses of participants in identifying the stimuli were interpreted in terms of attention, as an indication that sensory capture had occurred and, consequently, of whether participants were relying on either kinesthesis or touch.

This intrahaptic capture phenomenon is of interest because it suggests that conflict between intrahaptic sensory cues can go unnoticed; sometimes we are unaware of how we moved, and sometimes we do not know what we touched, or at least may not be certain. In the present study are four experiments that tested the idea that the effect observed by Symmons et al. (2007) is an example of sensory integration in the form of capture and, specifically, tested whether cutaneous information can dominate kinesthesis. In Experiment 1, we explored whether the effect observed by Symmons et al. (2007) occurs using stimuli simpler than letters and numbers (i.e., angles, which may make it easier to attend to movement than do complex shapes), and whether the removal of cutaneous stimuli (i.e., no raised-line or shear information) from one finger matters (i.e., when self-generated movement became the major cue for shape), a condition not present in Symmons et al.'s (2007) experiment. From the findings of Symmons et al. (2007), we hypothesized that this would result in cutaneous capture of kinesthesis. In Experiment 2, participants were instructed to endogenously direct attention to the kinesthetic stimulus (i.e., the sensory stimulus captured in Exp. 1) to control for response confusion and, indirectly, to explore the role of attention in this effect. Given the strength of the initial type of capture and the fact that it occurred when raised-line cutaneous input was removed, we hypothesized that tactile capture of kinesthesis would still occur, even with attention directed to the previously captured stimulus.

Experiment 3 explored the different roles that active and passively guided movement might play in intramodal haptic capture. We hypothesized that with passive movement, capture would be reduced because of (1) the diminished attenuation of reafference or (2) the attentional resources devoted to control in active movements being redeployed during passively guided movements to assess incoming stimuli. Finally, in Experiment 4, the raised-line information for the perceptually dominant finger was degraded in an attempt to change its weighting in sensory integration. We hypothesized that this would reduce the capture effect.

\section{Experiment 1}

The aim of Experiment 1 was to decouple cutaneous from kinesthetic stimulation. This was achieved by providing a 
cutaneous stimulus to one finger without kinesthetic information. Specifically, a raised line was yoked to the movement of the moving finger and presented against a stationary finger. The moving finger had kinesthetic stimulation with and without cutaneous information (i.e., the finger was moved by the participant, but the raised line was absent in some conditions). The trick was that, although the patterns were identical, each hand explored a different orientation of the pattern, potentially providing a different percept, as in the angles $<$ and $>$, for example (see Fig. 1). Based on the findings of Symmons et al. (2007), we hypothesized that cutaneous inputs alone (i.e., those from the stationary finger) would be weighted more heavily than kinesthetic inputs alone (i.e., those from the moving finger). As such, simply moving a raised-line stimulus under a stationary finger without kinesthetic information would be enough to result in a report of that angle in preference to the rotated version of the angle experienced at the moving fingertip when this finger was deprived of cutaneous input.

\section{Method}

Participants The data from 16 volunteers (i.e., university undergraduates and members of the general public) 18 to 46 years of age $(M=22.3$ years, $S D=7.1), 15$ of whom were

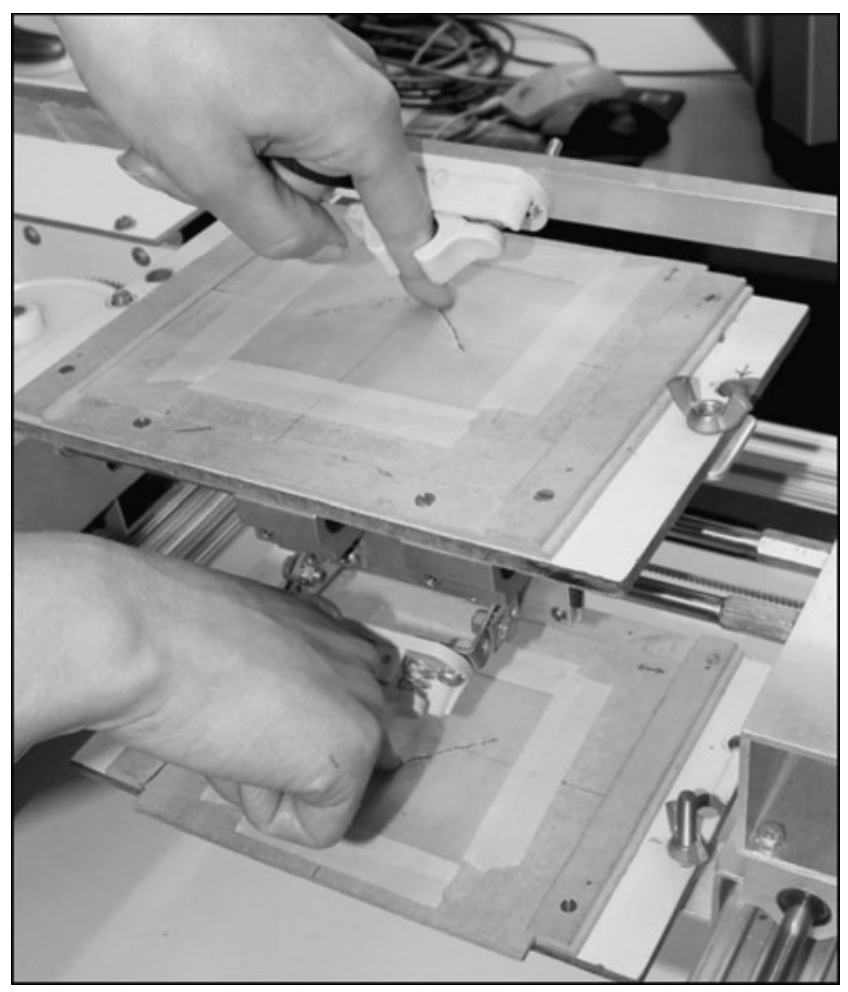

Fig. 1 The stimulus arrangement with the tactile display system. As the right index finger moves over the stationary $\vee$ stimulus (lower hand in the photograph), a $\wedge$ stimulus is moved underneath the stationary left index finger (upper hand) female and one male, were used. The responses of three additional participants (i.e., not counted among the 16 participants mentioned here) were removed prior to the analysis because of a failure to accurately identify one or more of the stimuli. Specifically, one participant felt that she was exploring circles, another felt as though the stimuli formed a straight line, and one felt that one of the angles was facing upward when, in fact, it was pointing to the right. None of the participants had any long-term or extensive experience with Braille, raised-line drawings, or tactile devices, nor did they report any motor or neurological abnormalities. All experiments presented here were carried out in accordance with the regulations of the Monash University Human Research Ethics Committee.

Materials Pairs of raised-line stimuli were prepared by drawing on sheets used in raised-line drawing kits in a manner similar to that of Magee and Kennedy (1980). Each arm of the raised-line stimulus was approximately $7.5 \mathrm{~cm}$ long, and the angle between the arms was $90^{\circ}$. The stimulus pairs were $>/<$ and $N / v$, where the two stimuli of each pair were identical but rotated $180^{\circ}$. A sheet of unscored raised-line paper was used for conditions in which no tangible line was present (i.e., the shear condition).

Apparatus The tactile display system (TDS) has two finger holders and two associated stimulus trays (see Fig. 1). The lower finger holder can be used to either passively guide a participant's finger over a stationary stimulus or, as was the case in this experiment, to grip a participant's finger while the participant actively explores a stationary stimulus. The upper finger holder keeps a finger stationary, while a stimulus is moved underneath it. The fingers are, on average, $\sim 16 \mathrm{~cm}$ from each other. The upper stimulus tray is attached to the lower finger holder so that they both move at the same time. However, if the lower finger moves in the shape of a $\mathrm{V}$, then, relative to the stationary upper finger holder, the upper stimulus traces out the shape of a $\wedge$. Owing to the physical construction of the device, the shape experienced by one finger is a $180^{\circ}$ rotation of what is felt at the other. Even so, each participant felt the raised line of any part of the stimulus at both fingers simultaneously. For example, in Fig. 1, the lower finger is at the intersection of the $\vee$. Simultaneously, the upper finger is at the intersection of the $\wedge$ - the corresponding position. If the next movement for the lower finger were to follow the line on the left-hand side of the $v$ to its endpoint (i.e., moving away from the body), the top finger would simultaneously sense the line on the right-hand side of the $\wedge$ to its endpoint.

The reader can experience the effect by tracing out one of the stimuli mentioned with the index finger of the left hand (i.e., let this finger move in space toward and away from your body) and placing a finger from their right hand, and 
keeping it stationary in space (i.e., it is always the same distance from the body and in front, say, of the body midline) on the center of the back of the left hand. If one does this, it follows that moving the left hand forward and to the left, with the index finger of the stationary right hand in contact with the top of it, the skin of the right index fingertip will feel a shear force similar to that which would occur if the right index finger moved across a stationary surface toward the body and to the right. The shear forces at the moving fingertip indicate motion in a $\vee$ trajectory, whereas the shear forces experienced at the stationary right fingertip indicate the inverse (i.e., a $\wedge$ ). Hence, a $\wedge$ on the upper display connected to one hand can be paired with its inverse on the lower display, connected to the other hand. These two shapes, one the inverse of the other, are the distal objects, and the issue in the present study was which of the distal shapes the observer detected.

Procedure The participants were shown the TDS and how the device worked in both upper (stationary finger holder) and lower (moving finger holder) modes prior to the commencement of testing. The participants were also shown how the device worked in the dual-finger mode (i.e., a moving finger in the lower finger holder can move a stimulus underneath a stationary finger in the top finger holder) but, as a stimulus was not placed in the upper tray, reference to $180^{\circ}$ rotations was avoided. Participants were also given an opportunity to feel a raised-line stimulus. At the conclusion of the instructions, participants were blindfolded and asked to place an index finger in each finger holder. They were told which finger (left or right) should be used in each finger holderthis first positioning of the fingers was counterbalanced across participants. This variable (i.e., finger positioning) was administered on a within-subjects basis and had two levels (i.e., left vs. right finger in the upper finger holder).

The participants were told that they would explore an angle with both fingers at the same time, but that one finger would be moving and the other would be stationary, with the stimulus moving underneath it. They were then told that their task was to identify "which way is the angle pointing?" by way of verbal report; no mention was made by the experimenter of the $180^{\circ}$ rotation of the stimuli. Participants were not directed to focus their attention on either hand in particular, nor were they given any indication that there might be a difference between the percepts arising from each hand. Each participant explored each pair of stimuli, with one of each pair in the upper tray and the other in the lower tray. Thus, each participant underwent a total of six trials in this experiment (i.e., a single presentation of each condition), examining the same pair of stimuli on each trial - the goal of which was to improve performance and avoid floor effects in a novel task. As an example, a participant got only a $\vee$ in the upper tray and its inverse in the lower tray; he or she would never get a different pair (e.g., a $\wedge$ in the upper tray and its inverse in the lower tray). Thus, stimulus combination was a between-subjects variable with four levels.

A separate variable (i.e., mode of delivery) was administered on a within-subjects, pseudorandomized basis. It had three levels:

1. Kinesthesis + Line + Shear: The participants could feel a line under both the active, participant-controlled moving index finger and the stationary index finger simultaneously.

2. Kinesthesis + Shear: The participants could feel a line under the stationary index finger but the active, participantcontrolled moving index finger could feel only a lightly textured plastic surface - there was no line but cutaneous shear forces (i.e., signals produced when the skin and a surface are laterally shifted in relation to each other) remained due to friction between the blank raised-line sheet and the fingertip. In this condition and the next, the feedback from the stationary finger (i.e., the finger in contact with the line) was relied upon to guide the action of the moving finger.

3. Kinesthesis: The participants could feel a line under the stationary index finger but the actively moving index finger received no cutaneous information-it moved through air only. Thus, the guide for the moving finger was the line in contact with the stationary finger. So, although the participant-controlled moving finger could move in any direction, it was guided by the contact between the stationary finger and the line. To clarify, the upper stimulus tray was attached to the lower finger holder so that they both moved at the same time. If the upper stimulus traced out the shape of a $\wedge$, the lower finger moved in the shape of a $\vee$ (see Table 1 for a full list of conditions, which were randomized within

Table 1 The variables and the levels of each variable of Experiment 1

\begin{tabular}{|c|c|c|}
\hline $\begin{array}{l}\text { Finger Positioning } \\
\text { (Within Subjects) }\end{array}$ & $\begin{array}{l}\text { Mode of Delivery } \\
\text { (Within Subjects) }\end{array}$ & $\begin{array}{l}\text { Stimulus Combination } \\
\text { (Between Subjects) }\end{array}$ \\
\hline $\begin{array}{l}\text { Right finger } \\
\text { stationary }\end{array}$ & $\begin{array}{l}\text { Kinesthesis }+ \\
\text { Line }+ \text { Shear }\end{array}$ & $\Lambda / \vee$ or $v / \wedge$ or $<>>$ or $>/<$ \\
\hline $\begin{array}{l}\text { Right finger } \\
\text { stationary }\end{array}$ & Kinesthesis + Shear & $\Lambda / \vee$ or $V / \wedge$ or $</>$ or $>/<$ \\
\hline $\begin{array}{l}\text { Right finger } \\
\text { stationary }\end{array}$ & Kinesthesis & $\Lambda / V$ or $V / \wedge$ or $</>$ or $>/<$ \\
\hline $\begin{array}{l}\text { Left finger } \\
\text { stationary }\end{array}$ & $\begin{array}{l}\text { Kinesthesis }+ \\
\text { Line }+ \text { Shear }\end{array}$ & $\Lambda / \vee$ or $v / \Lambda$ or $</>$ or $>/<$ \\
\hline $\begin{array}{l}\text { Left finger } \\
\text { stationary }\end{array}$ & Kinesthesis + Shear & $\Lambda / \vee$ or $v / \wedge$ or $<>$ or $>/<$ \\
\hline $\begin{array}{l}\text { Left finger } \\
\text { stationary }\end{array}$ & Kinesthesis & $\Lambda / \vee$ or $\vee / \wedge$ or $</>$ or $>/<$ \\
\hline
\end{tabular}

${ }^{a}$ A raised line was always present at the stationary finger. The details in this column outline the combination of stimuli present at the moving finger 
subjects). Though this is a complicated setup, all of the participants understood that they were required to use input to the stationary finger to control the moving finger, and thereby to cause the raised line to move under the stationary finger. They all maneuvered this stimulus smoothly and with ease.

In all conditions, the participants had control over the movement of the finger in the lower finger holder. In a bid to determine whether participants were aware of the discrepancy between what they experienced at the moving and stationary fingers, the participants were asked whether they had noticed anything odd at the completion of the experiment. Latencies were not recorded, as each participant was given sufficient time to provide a response, though this never took more than a minute. We debriefed participants at the end of the experiment.

\section{Results}

The primary question of interest was which of each stimulus pair the participant would name. As is shown in Table 2, nearly all participants reported the stimulus present at both fingers when the line was present under the moving finger; they made a statement akin to "under my left finger is an arrow pointing up, while under my right finger is an arrow pointing down." This suggested that kinesthetic and cutaneous stimuli together were as effective as a cutaneous stimulus alone. The vast majority of the participants reported the stimulus present at the stationary finger (i.e., the finger that received only cutaneous information) when the raised line was not present at the moving finger (i.e., when either kinesthesis + shear or kinesthesis only was presented at this finger), and thus they failed to notice the kinesthetic and/or shear stimuli present at the moving finger.

When cutaneous information was present at both the moving and stationary fingers (i.e., kinesthesis + line + shear in Table 2), the odds in favor of identifying both stimuli (i.e.,

Table 2 Percentages of the 16 participants (i.e., 16 data points per condition) reporting the stimuli at the stationary and/or moving fingers when stimulus pairs were displayed at these fingertips and when the raised line was available to one or both fingers

\begin{tabular}{lll}
\hline $\begin{array}{l}\text { Moving } \\
\text { Finger }\end{array}$ & Stimuli at Moving Finger & Origin of Response \\
\hline Right & Kinesthesis + Line + Shear & $100 \%$ both \\
Right & Kinesthesis + Shear & $88 \%$ stationary finger \\
Right & Kinesthesis & $100 \%$ stationary finger $^{\text {a }}$ \\
Left & Kinesthesis + Line + Shear & $94 \%$ both $^{\text {b }}$ \\
Left & Kinesthesis + Shear & $100 \%$ stationary finger \\
Left & Kinesthesis & $100 \%$ stationary finger \\
\end{tabular}

${ }^{\text {a }}$ One participant identified the moving stimulus, and another identified both. ${ }^{\mathrm{b}}$ One participant identified the moving stimulus the stimuli at the stationary and moving fingers) were significantly higher than the odds in favor of identifying both stimuli when a raised line was present at the stationary finger and kinesthesis + shear information was present at the moving finger (odds ratio $=960.99, z=3.92, p<.001$ ). In the latter condition, participants usually identified the shape of the cutaneous stimulus.

A second odds-ratio analysis found that the odds in favor of identifying both stimuli if the participant was provided with a stimulus composed of kinesthesis + line + shear at the moving finger were significantly higher than the odds in favor of identifying both stimuli in the kinesthesis-only condition (odds ratio $=3,100, z=7.81, p<.001$ ). Again, participants usually identified only the cutaneously presented angle.

When participants had a kinesthesis + shear stimulus at the moving finger, the likelihood of a response identifying both stimuli was not significantly higher than that of identifying both stimuli if the participant were provided with a kinesthetic-only stimulus (odds ratio $=3.00, z=0.77, p=.20$ ).

\section{Discussion}

Cutaneous capture of kinesthetic information was indicated in this experiment. Participants were significantly more likely to acknowledge their movements if those movements were paired with cutaneous information. However, the participants seemed unaware of the pattern of their self-generated finger movements when a raised line was present at the stationary finger and kinesthesis + shear or kinesthesis-only was present at the moving finger. The participants appeared to attribute the kinesthetic input (as secondary information) to the cutaneous input (which was primary for control, since it indicated whether one was on or off the target line).

The participants reported the directions in which both stimuli pointed when the raised line was present at both fingers. This contrasts with findings reported by Symmons et al. (2007), who found that only one stimulus was reported. Given that postexperiment questioning by Symmons et al. (2007) revealed that active explorers were aware of a discrepancy between the stimuli (although not necessarily of what the discrepancy was), it seems likely that their participants responded to what they deemed to be the most salient stimulus. The difference between Symmons et al.'s (2007) findings and those reported here may be due to the difference in stimuli. Symmons et al. (2007) used more complex letters and numbers, whereas simple angles were used here. We speculate that simple stimuli may ease cognitive load and allow attention to be focused on both stimuli in this condition.

It remains a possibility that response confusion confounded this effect. Perhaps, because of the instruction to identify the direction in which the arrow was pointing, participants mistakenly thought to primarily report the raised line and, consequently, reported the raised line at 
the stationary finger when no raised line was present at the moving finger. The next experiment was designed to address this concern about response confusion; a fortunate consequence of altering the instructions was that we were also able to assess the role that endogenous attention plays in intrahaptic capture.

\section{Experiment 2}

To assess whether response confusion had an impact on the extent of the cutaneous capture suggested in Experiment 1, the instructions given to the participants were changed so that attention was directed specifically to the movements of the moving hand, at the expense of the raised-line stimulus at the stationary hand. We reasoned that if we instructed participants to pay attention to their moving finger, the kinesthetic stimuli should be able to escape capture. However, given the strength of the capture in Experiment 1, we expected that tactile capture of kinesthesis would still occur. This experiment addressed a concern from Experiment 1 that participants might have mistakenly thought that they were only required to report the raised-line stimulus: If directing attention to the movement does not abolish the capture effect, then the effect would not be due to response confusion.

\section{Method}

Participants A group of 12 adults 19 to 66 years of age ( $M=$ 38.3 years, $S D=15.7)$ voluntarily participated; seven were women, five were men. These people were undergraduate students of Monash University or members of the general public who had not participated in Experiment 1.

Materials and apparatus The raised-line drawings produced for Experiment 1 were used here. Specifically, the stimuli were $>,<, \wedge$, and $\vee$. These stimuli were presented via the TDS in a manner consistent with kinesthesis-only conditions of Experiment 1 (i.e., a line at the stationary finger and movement only at the other finger).

Procedure The participants were shown the TDS and how the device worked in the dual-finger mode prior to the commencement of testing. However, reference to $180^{\circ}$ rotations was avoided, as a stimulus was not placed in the upper tray. The participants were shown a raised-line stimulus and told "you'll get something like this at the stationary hand and you'll use your other hand to move around it, but you won't feel a line at your moving finger." They were given an opportunity to touch a raised-line stimulus. The participants were directed to focus on their moving finger (i.e., "I want you to pay attention to what you're doing with your moving finger") and asked to "report how it moves" (see Table 3).
The participants were asked to place an index finger of their choosing in each finger holder ${ }^{1}$ at the conclusion of the instructions, and they were blindfolded.

The participants actively explored with a moving finger, simultaneously guiding a rotated stimulus under the stationary index finger of their other hand. As there was a risk that they might misunderstand the instructions, after participants responded to "report how it moves" they were asked "which finger was moving and which was the stationary finger?" This was an attempt to make sure that they were not misunderstanding the instructions to mean that they focus on the moving stimulus rather than the moving finger. After responding to this question, the participants were asked to remove the stationary finger from the upper finger holder of the TDS. With the moving finger still in the lower finger holder they were asked to "reproduce the movements you just made" (i.e., movements made to explore the cutaneous stimuli) with the bottom hand. If they responded incorrectly (i.e., produced a movement consistent with the direction the cutaneously presented angle was pointing in the upper tray), the participants were asked to reinsert their finger into the upper finger holder, explore the stimulus and "ignore the tactile information" (i.e., the stimulus in the upper tray). In this final condition, participants were also lightly tapped on the moving hand while this instruction was being given to further direct their attention to the moving hand. Each participant underwent four trials in Experiment 2 (one trial per condition) in the order outlined above. Again, they examined the same pair of stimuli on each trial (e.g., $<$ at the stationary finger, $>$ at the moving finger) and were debriefed at the end of the experiment.

\section{Results}

Table 3 shows that even with explicit instructions to focus on the movement path of the moving finger, most participants reported the stimulus present at the stationary finger.

Nine $(75 \%)$ of the 12 participants reported the direction of the cutaneous-only stimulus even when instructed to attend to the moving finger. If readers are surprised by these results, as we were, it may be because they are familiar with the literature demonstrating the dominance of kinesthesis over touch, or find it unlikely that cutaneous information could dominate. If so, they may hold an a priori probability in mind that, perhaps, we can make explicit. If we think about this experiment in a manner akin to an experiment assessing the Colavita effect, it is reasonable to assume that everybody would be able to identify both stimuli when those

\footnotetext{
${ }^{1}$ Pilot studies showed that the finger used in each tray did not alter the percentage of responses identifying the stimulus at either finger. As such, the participants were given the opportunity to use the finger that they deemed most appropriate/comfortable.
} 
Table 3 Percentages of the 12 participants (i.e., 12 data points per condition) reporting the stimuli at the stationary and moving fingers when cutaneous information was available at only the stationary finger

Question
Origin of Response (Stationary Finger)

$75 \%$

$0 \%$

$83 \%$

$50 \%$
Origin of Response (Moving Finger)

"I want you to pay attention to what you're doing with your moving finger" and "report how it moves"

"Which finger was moving and which was the stationary finger?"

"Reproduce the movements you just made"

"Re-explore the stimulus and ignore the tactile information"

In three of the four questions, the responses identified the distal shape. However, the question "Which finger was moving and which was the stationary finger?" required participants to provide a statement akin to "the finger closest to me is the moving finger" or "my right finger moves and my left finger stays still."

stimuli are presented independently of one another. For example, in Colavita experiments, participants can see the light and hear the sound when they are presented separately. Here, participants should be able to identify the directions in which the stimuli are pointing at the stationary and moving fingers when they are presented independently. As such, we have an a priori reason to assume that the percentage of people verbally identifying only the stimulus at the stationary hand would be close to $0 \%$, because participants should know how they moved. A binomial test with the test proportion set at .99 (i.e., they would correctly identify the kinesthesis-only condition $99 \%$ of the time) showed that participants were significantly likely to report the cutaneous-only stimulus ( $p<$ .001). A second binomial test revealed that, if there was a .99 probability that participants would reproduce movements consistent with the moving finger, they more often than not $(p<$ .001) produced movements consistent with the direction of the stimulus at the stationary finger (i.e., the finger receiving only cutaneous information). A third binomial test revealed that, if there was a .99 probability that people would identify the moving stimulus, there was a significant likelihood $(p<.001)$ that they would report the cutaneous-only stimulus when attention was verbally and physically (i.e., a light tap) directed toward the moving hand. Given these findings, we argue that cutaneous stimuli were weighted more heavily than kinesthetic stimuli, even with endogenous attention to the latter. As the effect was still present in this experiment, response confusion does not seem responsible for the capture effect.

When participants were verbally directed to pay attention to the moving finger (i.e., in response to the question "I want you to pay attention to what you're doing with your moving finger" and "report how it moves"), the odds favoring a response identifying the stimulus at the stationary finger (i.e., cutaneous-only) were, at best, marginally significantly different-between .1 and .05-from the odds in favor of identifying this stimulus in the verbal + physical orienting of attention condition (i.e., "Re-explore the stimulus and ignore the tactile information": odds ratio $=3.00, z=1.36, p=.07$ ). It seems that the addition of a physical cue (i.e., a tap on the hand) did not significantly alter the probability at which people would report the cutaneous stimulus.

We also wanted to compare the kinesthesis-only condition of Experiment 1 with the equivalent condition here, in an attempt to assess the influence of endogenous attention. When participants had kinesthesis-only information at the moving finger in Experiment 1, the odds favoring a response identifying the stimulus at the stationary finger (i.e., cutaneousonly) were significantly higher than the odds in favor of identifying the cutaneous-only stimulus in response to the "report how it moves" condition of Experiment 2 (odds ratio $=10.67$, $z=3.43, p<.001$ ), thus suggesting that attention has a modulating influence on cutaneous capture.

\section{Discussion}

The key finding to emerge from the analysis of the data of Experiment 2 was that participants made more cutaneous-only than kinesthesis-only responses on the bimodal trials. This finding provides an empirical demonstration of the existence of cutaneous capture of kinesthesis, which seems modulated, but not entirely determined, by attention, as demonstrated by the analysis comparing the results of Experiment 1 with those of Experiment 2. The participants were explicitly instructed to focus on the movement of the moving finger. This addressed the concern that participants might have misinterpreted the instructions of Experiment 1 as requesting a report of the cutaneous stimulus specifically. As the effect is still present even with instructions to focus on the moving finger, we assume that it is not an artifact of response confusion.

Very few prior studies have reported similar findings. In a study by Heller and Clyburn (1993), sighted, early-blind, and late-blind participants used their index fingers to feel embossed patterns consisting of large circles composed of small squares. Sighted participants tended to report both the large and small configurations, suggesting as much attention to the kinesthetic input (large circle) as to the cutaneous input (small squares), which may be thought 
of as competing in this situation. However, late-blind participants tended to report the small squares, suggesting more attention to, or possibly capture by, the cutaneous input. We also found that people were more likely to report the cutaneous input. An interesting question is why this result resembles that found in late-blind participants, and exactly how attention factors into this outcome.

Attention can modulate, but not explain, the Colavita effect (Spence, 2009) and does not seem to influence the ventriloquist effect (Bertelson, Vroomen, de Gelder, \& Driver, 2000). It is becoming clear, however, that attention and sensory integration interact in ways that are sensitive to many parameters in the chosen stimuli and their presentation (Talsma, Senkowski, Soto-Faraco, \& Woldorff, 2010). In our experiment, a type of inattentional blindness could be implicated; exogenous attention may have been captured by the cutaneous stimulus, even though endogenous attention was directed to kinesthesis. Cutaneous inputs may have captured attention because a small physical change at a body location (e.g., $300-\mathrm{Hz}$ oscillations at a fingertip) involuntarily pulls attention to that location, with the benefit that harmful stimuli can be effectively detected (Müller \& Giabbiconi, 2008). Perhaps the constant stimulation of the skin of the stationary fingertip, which has limited habituation to flutter-like stimulation (Müller \& Giabbiconi, 2008), dragged attention to that fingertip at the expense of attention to the moving hand.

This interpretation is complicated by the feedback element in our setup. It is difficult to argue that the movement was not attended to, given that the raised-line information was fed back from the stationary to the moving finger to guide movement, which suggests that some attentional resources must have been devoted to getting the movement correct, and this raises an interesting point. The effect presented here differs from most other inattentional blindness effects because it has this intermodal feedback element. Specifically, when there is no raised line at the moving finger, the angle felt at the stationary finger guides the active movement of the moving finger. The present study also introduced a perspective that may implicate attention in novel ways, because the cross-modal conflict is processed by feedback from the stationary finger to guide the moving finger's movement.

Attention seems to have had an effect on cutaneous capture, although bias was still present. This is encouraging, but the interpretation in terms of attention may be complicated by the feedback element in this task. Experiment 3 explored this aspect of the findings.

\section{Experiment 3}

Self-generated movements typically produce attenuation of the moving body part's afferent signal (Voss, Ingram,
Haggard, \& Wolpert, 2006). Hence, the feedback used to guide active exploration in this task might attenuate reafference from that movement and, thereby, facilitate cutaneous capture. In addition, the feedback element might engender a cognitive load that hinders endogenous attention to the movement, and thus also facilitates cutaneous capture. We thus used passively guided movements to assess whether the capture of kinesthesis seen in Experiment 1 was facilitated, at least in part, by the feedback element of the task and the attenuation of movement-related sensations. We thought that passively guided movements would reduce the percentage of responses, relative to Experiment 1, identifying the stimulus at the stationary finger. We also thought that, if participants were better able to identify their pattern of movement, they would be better able to "reproduce the movements just made."

\section{Method}

Participants The data from 12 volunteers (i.e., university undergraduates and members of the general public) 17 to 55 years of age $(M=26.9$ years, $S D=11.9)$ were used; ten of the volunteers were female, two were male. None had participated in Experiments 1 or 2.

Materials and apparatus The materials and apparatus were the same as those used in Experiment 2.

Procedure The participants were shown the TDS and how the device worked prior to the commencement of testing. There was no mention of $180^{\circ}$ rotations, and the instructions that the participants received were identical to those of Experiment 2. However, participants passively explored the stimuli with a moving finger (they relinquished control of their movement to the experimenter), while simultaneously experiencing a rotated stimulus under the stationary index finger of their other hand. The participants were directed to focus on their moving finger (i.e., "I want you to pay attention to your moving finger") and asked to "report how it moves" (see Table 4). After responding to "which finger was moving and which was the stationary finger?" participants were asked to remove the stationary finger from the TDS. Once again, with the moving finger still in the TDS's bottom finger holder, they were asked to actively "reproduce the movements just made" with this hand. As a final condition, the participants were asked to reinsert their finger into the upper finger holder, to explore the stimulus, and to "ignore the tactile information" (i.e., the stimulus in the upper tray). In this final condition, participants were lightly tapped on the moving hand while this instruction was being given to further direct their attention to the moving hand. The participants were debriefed at the end of the testing session. 
Table 4 Percentages of the 12 participants (i.e., 12 data points per condition) reporting the stimuli at the stationary, the moving, or both fingers when cutaneous information was available at only the stationary finger

\begin{tabular}{llll}
\hline Question & $\begin{array}{l}\text { Origin of Response } \\
\text { (Stationary Finger) }\end{array}$ & $\begin{array}{l}\text { Origin of Response } \\
\text { (Moving Finger) }\end{array}$ & $\begin{array}{l}\text { Origin of Response } \\
\text { (Both Fingers) }\end{array}$ \\
\hline $\begin{array}{l}\text { "I want you to pay attention to your moving finger" } \\
\text { and "report how it moves" }\end{array}$ & $50 \%$ & $0 \%$ & $50 \%$ \\
"Which finger was moving and which was the stationary finger?" & $0 \%$ & $100 \%$ & $0 \%$ \\
"Reproduce the movements you just made" & $58 \%^{\mathrm{a}}$ & $33 \%$ & $0 \%$ \\
"Re-explore the stimulus and ignore the tactile information" & $17 \%$ & $0 \%$ & $83 \%$ \\
\hline
\end{tabular}

${ }^{\text {a }}$ One participant was unable to reproduce the movement

\section{Results}

Table 4 shows that several participants identified both the moving and stationary stimuli in this experiment.

Six (50\%) out of 12 participants reported the direction of the cutaneous-only stimulus during passively guided movements, even when instructed to attend to the moving finger. A binomial test with the test proportion set at .99 (i.e., they would correctly identify the kinesthesis-only condition $99 \%$ of the time) showed that there was a significant chance that participants would still report cutaneous-only stimuli $(p<$ .001). When participants were asked to reproduce the movements they had made, and if we could expect $99 \%$ of the reproductions to mimic the direction of the stimulus at the moving finger, a significant number of participants produced movements consistent with the direction of the stimulus at the stationary finger $(p<.001)$. A third binomial test revealed that, if there was a .99 probability that people would identify the stimulus at the moving finger, there was a significant likelihood $(p=.006)$ that people would report the stimulus at the stationary finger (i.e., cutaneousonly) when attention was verbally and physically (i.e., a light tap) directed toward the moving hand.

When participants were verbally directed to pay attention to the passively moving finger, the odds favoring a response identifying the stimulus at the stationary finger (i.e., cutaneous-only) were significantly higher than the odds in favor of reporting the cutaneous-only stimulus for participants directed verbally and with a physical touch to the passively guided hand (odds ratio $=5.00, z=1.66, p=$ $.04)$. As such, it seems that attention and efference copy interact in ways that are sensitive to stimulus parameters, their presentation, and the instructions.

We also wanted to compare the kinesthesis-only condition of Experiment 1 with the "report how it moves" condition here in an attempt to assess the influence of efference copy. When participants had kinesthesis-only information at the moving finger in Experiment 1, the odds favoring a response identifying the stimulus at the stationary finger (i.e., cutaneous-only) were significantly higher than the odds in favor of identifying only this stimulus in the equivalent condition of Experiment 3 (odds ratio $=32.00, z=$ 2.97, $p=.001)$. In sum, an active, participant-controlled kinesthetic condition produced more reports of the raisedline form at the stationary finger than did a passively guided kinesthesis-only condition.

\section{Discussion}

Without feedback and attenuation of the motor command (i.e., efference copy present in self-generated movement), half of the participants remained unaware of the movements that they made. The other half were able to identify the stimuli presented to both hands (i.e., were aware of the movements that they made as well as the cutaneous stimulus). These results are different from those of Experiments 1 and 2.

It is surprising that participants in Experiment 1 were completely unaware of the way that they actively moved their finger, and given the comparison of these experiments, it seems likely that it was somehow related to the role of forward models in sensorimotor processes. In active (rather than passively guided) movement, forward models of the movement allow for prediction, and therefore attenuation, of the sensory (e.g., kinesthetic) consequences (Blakemore, Wolpert, \& Frith, 1998). The results from Experiment 1 may be the product of kinesthetic inputs being attenuated as they were well predicted by the forward model. As such, the salience of the cutaneous input was enhanced in these experiments because it could not be predicted.

Juravle and Spence (2011) showed that the execution of active movements during a juggling task suppresses taskirrelevant cutaneous inputs. They argued that comparison of the predicted and actual states attenuates the unnecessary sensory information (cutaneous inputs, in their experiments) and enhances the essential sensory information (kinesthetic information). Their finding is consistent with work (e.g., Hecht, Reiner, \& Karni, 2008; Seki, Perlmutter, \& Fetz, 2003) showing that self-generated movements increase the detection threshold of tactile stimuli presented on a moving body through nonspecific gating of incoming information. 
Gallace, Zeeden, Röder, and Spence (2010) argued that suppressing tactile stimuli during the execution of selfgenerated movements is advantageous, as it prevents potentially irrelevant stimuli (e.g., a fly landing on one's arm) from interfering with important movements (e.g., defending oneself from an attacker).

A crucial difference between earlier work and the research presented here seems to be that tactile stimuli were presented on the moving limb in earlier experiments. Here, tactile and kinesthetic stimulation arose at different hands and, as such, our findings seem analogous to those of Abbruzzese, Ratto, Favale, and Abbruzzese (1981) in that, although object form is provided by kinesthetic information, we do not notice these movements because of gating at particular levels (e.g., spinal). Instead, we notice the features of the objects (Stoeckel et al., 2004). Additionally, in our study the captured stimulus was not task-irrelevant, as the tactile stimulus was in Juravle and Spence's (2011) juggling task. As such, it was less advantageous to use prediction to gate for it.

It is also possible that, in this task, the removal of the feedback element itself is a factor in diminishing cutaneous capture, apart from the consequences for forward modeling. It may be that passive movement conditions decrease the task load so much that attention is more easily directed to the path of the moving hand, an interpretation consistent with Magee and Kennedy (1980), who suggested that the attentional capacity of each individual's haptic modality was overburdened by the difficulty associated with the planning and monitoring of active movements.

The absence of feedback and attenuation of the motor command had an effect on cutaneous capture. However, the interpretation in terms of weighted cue integration may be complicated by the reliability of the cutaneous sensory input. Experiment 4 explored this aspect of the findings.

\section{Experiment 4}

The fourth experiment explored the influence of increasing noise in the cutaneous signal - that is, placing a rubber thimble over the fingertip of each participant that made the ridges of the raised lines less discernible, and thereby allowed kinesthetic information to be weighted more heavily (cf. Loomis \& Lederman, 1986). Chabris, Weinberger, Fontaine, and Simons (2011) suggested that "one hallmark of inattentional blindness is that increasing the effort required by the primary task decreases noticing of unexpected events" (p. 152). As such, if we were to increase task load by degrading the raised line, we should get more kinesthetic blindness if this is an inattentional blindness effect. On the other hand, if we were to degrade the raised line and the capture was reduced, a plausible conclusion would be that it is more like a sensory integration (cue capture) effect.

\section{Method}

Participants The data from 12 volunteers 18 to 51 years of age $(M=30.8$ years, $S D=13.3)$ were used; ten volunteers were female and two male. These people were either undergraduates or members of the general public, and none had participated in Experiments 1-3. The responses of two further participants were removed prior to analysis because of a failure to accurately identify one or more of the stimuli. Specifically, although both of these people could identify that their movement was a $180^{\circ}$ rotation of what they were feeling, they felt as though the stimulus that they were exploring was pointing to the right (or left) when in fact it was pointing upward; it may be that these participants were focusing on just one arm of the arrowhead.

Materials, apparatus, and procedure The stimuli, materials, and procedure used here were the same as those used in Experiment 2, with the difference that participants were presented with degraded versions of the raised-line stimuli (i.e., a rubber thimble was placed over the stationary index finger).

\section{Results}

Table 5 shows that even with explicit instructions to focus on the moving finger and degraded cutaneous information, most participants reported the stimulus present at the stationary finger.

Eleven people (92\%) of the 12 reported the direction of the cutaneous-only stimulus, even with degraded cutaneous

Table 5 Percentages of the 12 participants (i.e., 12 data points per condition) reporting the stimuli at the stationary and moving fingers when cutaneous information was available at only the stationary finger

Question
Origin of Response

(Stationary Finger)

$92 \%$

$0 \%$

$75 \%$

$25 \%$
Origin of Response (Moving Finger)

"I want you to pay attention to what you're doing with your

moving finger" and "report how it moves"

"Which finger was moving and which was the stationary finger?"

"Reproduce the movements you just made"

"Re-explore the stimulus and ignore the tactile information"
$8 \%$

$100 \%$

$25 \%$

$75 \%$ 
information and instructions to attend to the moving finger. A binomial test with the test proportion set at .99 showed that participants were likely to report the cutaneous-only stimulus $(p<.001)$. A second binomial test revealed that, if we assume that there was a .99 probability that participants would reproduce movements consistent with the moving finger, they were likely $(p<.001)$ to produce movements consistent with the direction of the stimulus at the stationary finger (i.e., the finger receiving only cutaneous information). A third binomial test revealed that, if there was a .99 probability that people would identify the moving stimulus, there was a significant likelihood ( $p<.001)$ that they would report the cutaneous-only stimulus when attention was directed verbally and with a physical touch to the moving hand.

When participants were verbally directed to pay attention to the moving finger (i.e., responses to the question "I want you to pay attention to what you're doing with your moving finger" and "report how it moves"), the odds favoring a response identifying the direction of the stimulus at the stationary finger (i.e., cutaneous only) were significantly higher than the odds in favor of reporting this stimulus in the verbal + physical orienting of attention condition (i.e., "Re-explore the stimulus and ignore the tactile information": odds ratio $=33.33, z=2.83, p=.002$ ). As such, it seems that attention and the precision of information interact in ways that are sensitive to stimulus parameters, their presentation, and the instructions.

To further examine how degrading the cutaneous stimulus affected cutaneous capture, the responses given in the kinesthesis-only condition of Experiment 1 and the "report how it moves" condition of Experiment 4 were compared directly. The analysis revealed that there was no significant difference between the odds in favor of responding to the cutaneous-only stimulus in the kinesthesis-only condition of Experiment 1 and the odds favoring a response identifying the cutaneous-only stimulus in the equivalent condition of Experiment 4 (odds ratio $=2.91, z=0.73, p=.23$ ). This finding suggests that the magnitude of the effect was not significantly influenced by degrading the cutaneous stimulus (see Fig. 2).

\section{Discussion}

Cutaneous capture trended higher relative to Experiments 2 and 3, and was not significantly different from Experiment 1, where Experiments 2 and 3 had been. Linear, weighted cue integration does not seem to be at play since, in that case, the capture should have decreased with decreasing stimulus reliability. It may be that the thimble forced participants to increase attention to the cutaneous stimulus, which may have contributed to a type of inattentional blindness of the kinesthetic stimulus. Further studies will be needed to interpret the effects of degrading stimulus strength.

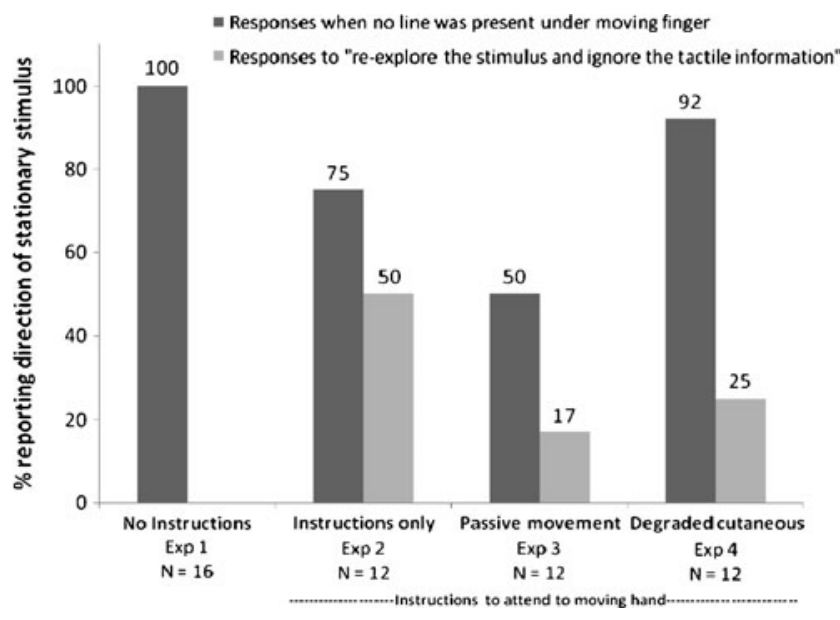

Fig. 2 Results of Experiments 1-4: Percentages of participants in each condition who reported only the stimulus presented to the stationary finger

There seems to be an interaction such that the effect can be reversed across conditions (see Fig. 2). When attention is firmly on the moving finger ("re-explore the stimulus and ignore the tactile information"), the thimble has little impact on the ability to identify the stimulus at the moving finger, but when attention is less firmly on the moving finger ("report how it moves"), degraded stimulus information plays much more of a role. Perhaps degrading the tactile stimulus made it easier to shift attention to the moving finger.

\section{General discussion}

Despite attempts to impede it, cutaneous capture of kinesthetic information occurred in all of the experiments, but was very strong in Experiments 1 and 4. In order to explore this phenomenon, attention was endogenously directed to the captured kinesthetic information in Experiment 2, which weakened but certainly did not abolish the capture effect. Experiment 3 showed that passive movements that do not involve feedback between cues, or a copy of the motor command present in selfgenerated movement, reduced the extent of capture relative to that found in Experiment 1, but again, these did not abolish the effect. Finally, Experiment 4 indicated that degrading the cutaneous signal at the stationary finger did not significantly modify cutaneous capture, relative to Experiment 1.

There is some inherent ambiguity in most perceptual situations, which means that the brain is often required to integrate sensory input and action into a robust experience of the world (Ernst \& Bülthoff, 2004). It has been suggested that in order to integrate information from multiple (sub) modalities, the brain uses a linear weighting rule in which the weights are determined by the signal's estimated reliability relative to other available sources (Newell, 2004; Sun, Campos, \& Chan, 2004). The brain combines sensory information in a way that attempts to minimize uncertainty 
(variance) in the final estimate, and thus produce a more dependable percept (Helbig \& Ernst, 2007; Wada, Kitagawa, \& Noguchi, 2003). It may be that with the line absent at the moving finger, some ambiguity (or conflict) was present between cues. In this situation, most participants reported the shape at the stationary finger (in Exp. 1) and did not report having noticed a discrepancy. It seems possible that this was an example of cutaneous capture of kinesthesis in which the weaker - or perhaps less attention-getting-stimulus was perceived as being identical to that arising from the dominant sensory input.

It is relevant here that the results of Experiment 4 suggest that optimal cue integration is not straightforwardly the mechanism behind this effect. As mentioned earlier, Bergmann Tiest et al. (2011) demonstrated that for perception of traversed distance, the discrimination threshold of estimates based on cutaneous information was larger than that for those based on kinesthetic inputs. Furthermore, these authors found that cue combination relied predominantly on kinesthetic information, with no obvious connection to optimal cue integration. This corresponds well with the present study, with the exception that cue reliance went in the other direction here (i.e., cutaneous cues seemed to capture kinesthetic cues). This difference may be explained by the stimuli and task: For shape detection, cutaneous input at the finger pad might be more reliable than kinesthetic information, and vice versa for traversed-distance estimation.

It is possible for attention to generate dramatic perceptual changes (Chabris et al., 2011). In the effect presented here, attention seems to be a contributing factor, since, as was shown in Experiment 2, the capture effect was diminished with endogenous attention directed to the captured kinesthetic stimulus. With regard to exogenous attention-grabbing by the more precise stimulus, it would be expected that decreasing the precision of the cutaneous stimulus with the thimble, in Experiment 4, should decrease the capture effect, but this did not happen. The interplay between attention and multisensory integration is complex (Talsma et al., 2010), and further studies are needed to explore this issue.

Pritchett et al. (2011) demonstrated tactile change blindness. Their participants were not aware of the location of the tactile change but were aware that a change had occurred. In the effect presented here, participants knew that they had moved but were oblivious as to how they had moved — ongoing incoming somatosensory inputs were used to guide ongoing movements, and perceived movement appeared to fit the cutaneous input. This is striking because it shows that input from stimuli in the external world, such as felt angles, can determine how we perceive our own movement, even when we are fully aware, and in control, of the movement.

Theoretically, this interpretation in terms of attention and active versus passively guided movement raises three interesting questions. First, what does the Bayesian brain theory associated with optimal integration predict about how sensory integration differs between passive versus active movement? Specifically, how does the Bayesian brain hypothesis predict patterns of sensory capture under such conditions? Second, how does the Bayesian brain theory relate to attention? Specifically, how is sensory integration modulated by attention, according to this theory? Third, how does passive versus active movement interact with attention? Specifically, does endogenous attention to some sensory cues facilitate or hinder perception of them? These are questions for further theorizing and experimentation. We note that all three questions are dealt with together within a generalization of the Bayesian brain theory associated with predictive coding and free-energy minimization (Friston, 2010). In particular, active movement allows for better prediction, and hence less prediction error, of own movement, which would facilitate perceptual suppression of kinesthesis; furthermore, the framework treats attention as optimization of the precision of prediction error, suggesting that increases in attention should counteract perceptual suppression (Feldman \& Friston, 2010).

Although haptics is composed of separate elements that usually work together, these elements can be teased apart in the laboratory using devices such as the TDS (Symmons, Richardson, \& Wuillemin, 2008). This is of relevance to the multitude of modern industrial and medical applications employing mechanical interfaces with haptic feedback, all of which are under real-time control, because conflict between intrahaptic sensory stimuli can go unnoticed. Our results cast doubt on the idea that kinesthetic information is somehow more important than cutaneous information. Although Juravle and Spence (2011) established "movement-related tactile sensory suppression" (p. 87), the result of teasing these haptic elements apart seems to indicate that tactile sensation may decrease awareness of movement parameters. The experiments reported here suggest that this cutaneous capture of kinesthesis is modulated by externally generated movement.

Author note The authors would like to thank John Kennedy for helpful comments.

\section{References}

Abbruzzese, G., Ratto, S., Favale, E., \& Abbruzzese, M. (1981). Proprioceptive modulation of somatosensory evoked potentials during active or passive finger movements in man. Journal of Neurology, Neurosurgery, and Psychiatry, 44, 942-949.

Bergmann Tiest, W. M., van der Hoff, L. M. A., \& Kappers, A. M. L. (2011). Proceedings of the IEEE World Haptics Conference (pp. 593-597). Piscataway, NJ: IEEE Press.

Bertelson, P., Vroomen, J., de Gelder, B., \& Driver, J. (2000). The ventriloquist effect does not depend on the direction of deliberate visual attention. Perception \& Psychophysics, 62, 321-332. doi:10.3758/BF03205552 
Blakemore, S.-J., Wolpert, D. M., \& Frith, C. D. (1998). Central cancellation of self-produced tickle sensation. Nature Neuroscience, 1, 635-640.

Calabro, F. J., Soto-Faraco, S., \& Vaina, L. M. (2011). Acoustic facilitation of object movement detection during self-motion. Proceedings of the Royal Society B, 278, 2840-2847.

Chabris, C. F., Weinberger, A., Fontaine, M., \& Simons, D. J. (2011). You do not talk about Fight Club if you do not notice Fight Club: Inattentional blindness for a simulated real-world assault. $i$-Perception, 2, 150-153.

Colavita, F. B. (1974). Human sensory dominance. Perception \& Psychophysics, 16, 409-412.

Dodge, R. (1900). Visual perception during eye movement. Psychological Review, 7, 454-465.

Ernst, M. O. (2004). Multisensory integration: A late bloomer. Current Biology, 18, R519-R521.

Ernst, M. O., \& Banks, M. S. (2002). Humans integrate visual and haptic information in a statistically optimal fashion. Nature, 415 , 429-433. doi:10.1038/415429a

Ernst, M. O., \& Bülthoff, H. H. (2004). Merging the senses into a robust percept. Trends in Cognitive Sciences, 8, 162-169. doi:10.1016/ j.tics.2004.02.002

Feldman, H., \& Friston, K. J. (2010). Attention, uncertainty, and freeenergy. Frontiers in Human Neuroscience, 4, 215. doi:10.3389/ fnhum. 2010.00215

Friston, K. (2010). The free-energy principle: A unified brain theory? Nature Reviews Neuroscience, 11, 127-138.

Gallace, A., Zeeden, S., Röder, B., \& Spence, C. (2010). Lost in the move? Secondary task performance impairs tactile change detection on the body. Consciousness and Cognition, 19, 215-229. doi:10.1016/j.concog.2009.07.003

Hecht, D., Reiner, M., \& Karni, A. (2008). Enhancement of response times to bi- and tri-modal sensory stimuli during active movements. Experimental Brain Research, 185, 655-665.

Helbig, H. B., \& Ernst, M. O. (2007). Optimal integration of shape information from vision and touch. Experimental Brain Research, 179, 595-606. doi:10.1007/s00221-006-0814-y

Heller, M. A., \& Clyburn, S. (1993). Global versus local processing in haptic perception of form. Bulletin of the Psychonomic Society, $31,574-576$

Juravle, G., \& Spence, C. (2011). Juggling reveals a decisional component to tactile suppression. Experimental Brain Research, 213, 87-97.

Loomis, J. M., \& Lederman, S. J. (1986). Tactual perception. In K. Boff, L. Kaufman, \& J. Thomas (Eds.), Handbook of perception and human performance (Vol. 2, pp. 1-41). New York, NY: Wiley.

Magee, L. E., \& Kennedy, J. M. (1980). Exploring pictures tactually. Nature, 283, 287-288.

Müller, M. M., \& Giabbiconi, C. M. (2008). Attention in sense of touch. In M. Grunwald (Ed.), Human haptic perception: Basics and applications (pp. 199-206). Boston, MA: Birkhäuser Verlag.
Newell, F. N. (2004). Cross-modal object recognition. In G. Calvert, C. Spence, \& B. Stein (Eds.), The handbook of multisensory processes (pp. 123-140). Cambridge, MA: MIT Press.

Pick, H. L., Warren, D. H., \& Hay, J. C. (1969). Sensory conflict in judgements of spatial direction. Perception \& Psychophysics, 6, 203-205.

Pritchett, D., Gallace, A., \& Spence, C. (2011). Implicit processing of tactile information: Evidence from the tactile change detection paradigm. Consciousness and Cognition, 20, 534-546.

Sanabria, D., Soto-Faraco, S., \& Spence, C. (2004). Exploring the role of visual perceptual grouping on the audiovisual integration of motion. NeuroReport, 15, 2745-2749.

Seki, K., Perlmutter, S. I., \& Fetz, E. E. (2003). Sensory input to the primate spinal cord is presynaptically inhibited during voluntary movement. Nature Neuroscience, 6, 1309-1316.

Seno, T., Ito, H., \& Sunaga, S. (2011). Inconsistent locomotion inhibits vection. Perception, 40, 747-750.

Sinnett, S., Spence, C., \& Soto-Faraco, S. (2007). Visual dominance and attention: The Colavita effect revisited. Perception \& Psychophysics, 69, 673-686. doi:10.3758/BF03193770

Spence, C. (2009). Explaining the Colavita visual dominance effect. Progress in Brain Research, 176, 245-258.

Stoeckel, M. C., Weder, B., Binkofski, F., Choi, H. J., Amunts, K., Pieperhoff, P., . . . Seitz, R. J. (2004). Left and right superior parietal lobule in tactile object discrimination. European Journal of Neuroscience, 19, 1067-1072.

Sun, H. J., Campos, J. L., \& Chan, G. S. (2004). Multisensory integration in the estimation of relative path length. Experimental Brain Research, 154, 246-254.

Symmons, M. A., Richardson, B. L., \& Wuillemin, D. B. (2007). Intrasensory attention: Kinaesthetic versus cutaneous inputs. Perception, 36, 880-887. doi:10.1068/p5690

Symmons, M. A., Richardson, B. L., \& Wuillemin, D. B. (2008). Components of haptic information: Skin rivals kinaesthesis. Perception, 37, 1596-1604. doi:10.1068/p5855

Talsma, D., Senkowski, D., Soto-Faraco, S., \& Woldorff, M. G. (2010). The multifaceted interplay between attention and multisensory integration. Trends in Cognitive Sciences, 14, 400-410. doi:10.1016/ j.tics.2010.06.008

van Beers, R. J., Wolpert, D. M., \& Haggard, P. (2002). When feeling is more important than seeing in sensorimotor adaptation. Current Biology, 12, 834-837.

Voss, M., Ingram, J. N., Haggard, P., \& Wolpert, D. M. (2006). Sensorimotor attenuation by central motor command signals in the absence of movement. Nature Neuroscience, 9, 26-27.

Wada, Y., Kitagawa, N., \& Noguchi, K. (2003). Audio-visual integration in temporal perception. International Journal of Psychophysiology, 50, 117-124.

Ziat, M., Hayward, V., Chapman, C., Ernst, M., \& Lenay, C. (2010). Tactile suppression of displacement. Experimental Brain Research, 206, 299-310. 